Nota de investigación

\title{
Propiedades dimensionales de las mazorcas para el diseño de máquinas
}

\author{
Eugenio Romantchik Kriuchkova \\ Erubiel Bernabe López ${ }^{\S}$ \\ Pedro Cruz Meza \\ José Guillermo Cebada Reyes \\ Noé Velázquez López \\ Posgrado en Ingeniería Agrícola y Uso Integral del Agua-Universidad Autónoma Chapingo. Carretera \\ México-Texcoco km 38.5. Chapingo, Estado de México, México. CP. 56230. \\ (eugenio.romantchik@gmail.com; $\quad$ guillermo.cebada@gmail.com; $\quad$ pcruzmeza@yahoo.com.mx; \\ noe.velazquez@gmail.com). \\ ${ }^{\S}$ Autor para correspondencia: hades.eruy@gmail.com.
}

\section{Resumen}

Para diseñar máquinas cortadoras y deshojadoras de mazorcas (Zea mays L.), es necesario conocer las propiedades geométricas del fruto que forman los datos de entrada para la evaluación de los modelos matemáticos del sistema fruto-máquina. Se determinaron tres características geométricas nuevas: diámetro de corte óptimo (do), longitud de la base al diámetro mayor (lbdm) y longitud de la base al diámetro de corte óptimo (lbco) de una muestra de 50 mazorcas de maíz blanco de la variedad Murano, del estado de Morelos, así como longitud total (lt) y diámetro mayor (dm). Del mismo modo para una muestra de 103 mazorcas variedad Arroz del estado de Veracruz se determinaron $1 \mathrm{bco}, \mathrm{lbdm}$, dm y lt, además se realizó un registro fotográfico y se procesó con el software OpenCV con determinación de dos parámetros más-área (A) y longitud de la base al centroide (lbc). El análisis estadístico mostró que existe una correlación simple de 0.905 entre lbcolbdm y dos coeficientes de correlación múltiple de 0.904 entre dm-lbdm-lbco, y de 0.917 entre ltlbdm-lbco para la variedad Murano. La variedad Arroz mostro una correlación múltiple de 0.66 entre lbco-dm-lbdm-lt-A-lbc. Con los resultados se establecieron diferentes modelos matemáticos de relación entre los parámetros de la mazorca para su aplicación en el diseño de un prototipo mejorado de deshojadora.

Palabras clave: Zea mays L. correlación, mazorca, parámetros geométricos.

Recibido: julio de 2019

Aceptado: agosto de 2019 
El maíz (Zea mays) representa la mitad del volumen total de alimentos que se consumen cada año y proporcionar a la población cerca de la mitad de las calorías requeridas. En 2006, la producción ascendió a 21.3 millones de toneladas, en tanto que la demanda fue de 26.2 millones de toneladas, por lo que se trata de aprovechar algunas de las partes de la planta como la hoja o totomoxtle para diferentes usos (Castañeda Zavala, 2007).

La beneficiadora Agroindustrias del Río clasifica la hoja cortada en siete categorías y presentaciones, cuanto más grande sean la longitud y ancho de las hojas la calidad es mayor. Esto depende de la variedad de maíz y el método de corte, que pueden ser en forma manual, deshojado manual con ayuda de un banco con disco dentado y extracción mecanizada de la hoja. Maquinas industriales cortan parcialmente las mazorcas en la parte basa y final para un deshojado posterior; la calibración previa que no permite alteraciones de la distancia de corte de los extremos de la mazorca (Rossi \& McGuire, 1992).

El Institutito Tecnológico Superior de Teziutlán desarrollo un prototipo accionado por pedal que funciona como guillotina (Rodríguez-Ventura, 2010) pero la posición de las cuchillas ocasiona que la hoja no tenga un corte uniforme. Otra máquina que realiza la separación de las hojas con un rendimiento de 790 mazorcas $\mathrm{h}^{-1}$ genera daños de un máximo de $3.41 \%$ a las hojas y $53.35 \%$ de grano desperdiciado con una media de 10.94\% (Cruz-Meza et al., 2010).

Estos valores indican que la máquina tiene deficiencias relacionadas con la geometría variable de las mazorcas. Las consideraciones de las propiedades dimensionales son factores para tomar en cuenta en el diseño de una máquina con un objetivo particular (Pérez-López et al., 2009), como lo es obtener hojas de mayor calidad. Para optimar el proceso de corte, Romantchik et al. (2016) describe una metodología para ajustar el disco de corte de una máquina deshojadora de mazorcas para tamal mediante el procesamiento de imágenes para calcular la longitud de corte optima (lbco).

Hace uso de este parámetro para calcular con una tarjeta de procesamiento la distancia del centro de una cámara al corte optimo (lcam), conociendo el ancho de trabajo de la cámara (atc), se localiza la posición de la mazorca como $\mathrm{x}_{0}$ que al sumarle lbco se convierte en la distancia $\mathrm{x}_{1}$. Con estos valores se aplica la ecuación (1), para determinar la distancia de corte de la mazorca al origen del dispositivo (dv), restando lcam a la distancia prestablecida del origen al centro de la cámara (lcc) con la ecuación (2) y calcula la distancia de ajuste (da) del disco de corte con la ecuación (3). Este dispositivo está en desarrollo en la Universidad Autónoma Chapingo (Bernabe, et al., 2018).

$\operatorname{lcam}=\frac{\text { atc }}{2}-\mathrm{x}_{1}$

$\mathrm{dv}=\mathrm{lcc}-\mathrm{lcam}$

$\mathrm{da}=\mathrm{dv}-\mathrm{dc}$

Para mejorar opciones de venta y diseño de las máquinas en la industria agroalimentaria se usan las propiedades físicas, mecánicas, biológicas, térmicas, etc. de los productos agrícolas, así como sus modelos matemáticos creados a partir de dichas características (Prado-Martínez et al., 2012). Las características agronómicas de las mazorcas son generalmente determinados como indicadores de productividad (Ávila et al., 2009). Se han analizado diferentes parámetros de las mazorcas de maíces nativos entre ellos: longitud, ancho, cantidad de hileras e índice ancho entre longitud con el propósito de determinar la calidad de elote (Ortiz-Torres, et al., 2013). 
Se caracterizaron química y morfológicamente las hojas de maíz y las propiedades biométricas (longitud y diámetro) para producir papel (Prado-Martínez et al., 2012). El conocimiento de las propiedades físico-mecánicas durante la evaluación de modelos matemáticos de la dinámica del sistema fruto-pedúnculo se precisa, para poder optimizar los regímenes de trabajo de las máquinas (Bouza et al., 2006).

Asimismo, el uso de un sistema de visión artificial proporciona un conjunto de funciones de análisis de patrones e imágenes, proporcionando atributos numéricos de los objetos o escenarios que se examinan (Velázquez-López et al., 2011), permitiendo la caracterización de las propiedades dimensionales de manera más precisa y rápida. Se ha utilizado el análisis de imágenes circulares de coco para determinar dos parámetros, la circularidad y el diámetro de Feret (Ramírez-Ramírez y Hahn-Schlam, 2013), para predecir el lugar del corte y optimizar la extracción de la pulpa en coco (Hahn-Schlam et al., 2012).

En este trabajo se determinaron diferentes modelos para calcular la distancia desde la base al corte óptimo para mazorcas. Esto permitirá mejorar el diseño de máquinas deshojadoras de mazorcas al implementar un modelo para cortar el pedúnculo de manera precisa, para que todas las hojas queden libres y se puedan deshojar rápidamente sin rasgarlas, con lo que se evita el desperdicio de granos, que se limita a sólo una hilera de granos por mazorca.

El experimento se realizó durante el mes de noviembre de 2015 con una muestra de 50 mazorcas de maíz blanco de la variedad hibrida Murano, procedentes del estado de Morelos. Las mediciones se llevaron a cabo con un Vernier digital marca Truper ${ }^{\circledR}$ con una tolerancia de $\pm 0.05 \mathrm{~mm}$, una cinta métrica Pretul ${ }^{\circledR}$ con una tolerancia de $\pm 0.1 \mathrm{~mm}$ y un calibrador de alturas marca Mitutoyo con escala milimétrica de $300 \mathrm{~mm}$ con una precisión de $\pm 0.05 \mathrm{~mm}$, en el laboratorio de metrología del Departamento de Ingeniería Mecánica Agrícola de la Universidad Autónoma Chapingo.

Las dimensiones geométricas se midieron en una sola vista superior (Figura 1). Se determinó la longitud total de cada mazorca (lt), el diámetro mayor ( $\mathrm{dm})$ y la longitud de la base a esta (lbdm). La longitud de la base a la zona de corte óptimo (lbco) y el diámetro de corte óptimo (do), se realizó cortando cada mazorca de manera transversal para visualizar el pedúnculo y medirlo con la cinta métrica.

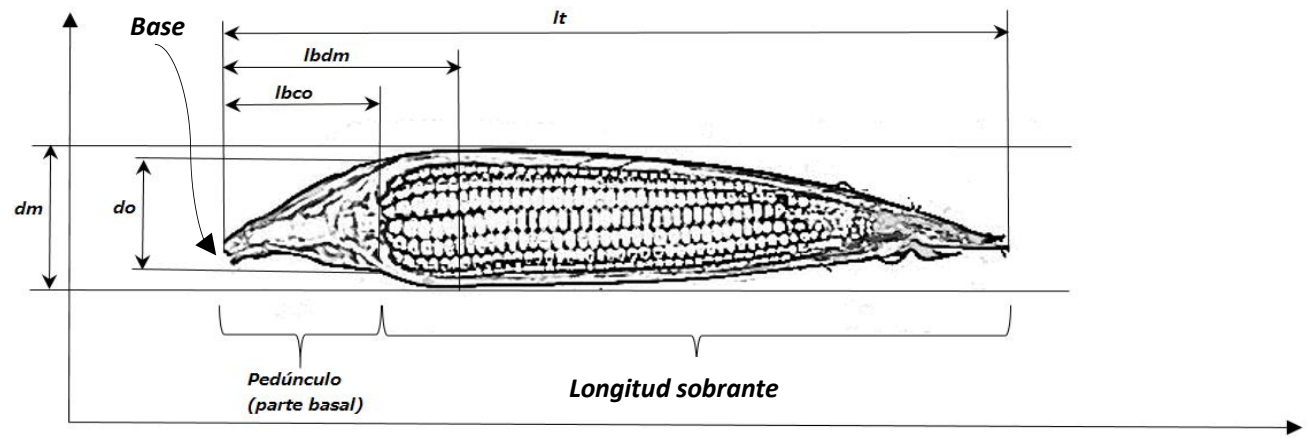

Figura 1. Parámetros geométricos medidos en mazorcas de la variedad Murano. 
Con el módulo de pruebas de estadística del paquete Microsoft Excel versión 2013 se realizó el análisis estadístico (Cuadro 1). Se estableció que para que la correlación fuese significativa, el coeficiente de correlación de Pearson (r), debía cumplir la siguiente condición: $r \geq 0.65$. Se efectuaron diez combinaciones de dos variables con cinco parámetros. Se hizo un análisis de varianza para determinar la existencia de un grado de correlación (Infante \& Zárate de Lara, 1984).

Cuadro 1. Estadísticas descriptivas de los parámetros geométricos de las mazorcas variedad Murano.

\begin{tabular}{|c|c|c|c|c|c|}
\hline \multirow{2}{*}{ Parámetros } & lbco & lt & $\mathrm{lbdm}$ & $\mathrm{dm}$ & do \\
\hline & \multicolumn{3}{|c|}{$(\mathrm{cm})$} & \multicolumn{2}{|c|}{$(\mathrm{mm})$} \\
\hline Media & 12.7 & 35.38 & 14.8 & 65.95 & 62.12 \\
\hline Mediana & 12.45 & 35.75 & 14.65 & 66.26 & 62.03 \\
\hline Desviación estándar & 2.21 & 2.68 & 2.29 & 2.67 & 2.44 \\
\hline Varianza & 4.9 & 7.21 & 5.25 & 7.13 & 5.96 \\
\hline Rango & 10.7 & 11.5 & 9.9 & 11.83 & 13.91 \\
\hline Mínimo & 7.9 & 30 & 9.9 & 59.8 & 52.69 \\
\hline Máximo & 18.6 & 41.5 & 19.8 & 71.63 & 66.6 \\
\hline
\end{tabular}

Se valoró la ecuación obtenida con del error absoluto. Se realizaron diez combinaciones de tres variables con cinco parámetros y se calcularon los coeficientes correlación múltiple (R) y el coeficiente de determinación $\left(\mathrm{R}^{2}\right)$. Se estableció que para una correlación significativa se debía cumplir la siguiente condición, $\mathrm{R} \geq 0.9$. Se llevó a cabo un análisis de varianza de las variables cuyas combinaciones cumplieron la condición antes mencionada. Para las combinaciones que cumplieron este criterio se buscó relacionarlas mediante una regresión lineal. De los coeficientes obtenidos de establecieron los modelos lineales.

El procedimiento usado para combinar cinco parámetros de tres variables se repitió para cuatro y cinco variables. Con una muestra de 103 mazorcas de maíz de la variedad criolla Arroz recolectadas de manera aleatoria en el municipio de Papantla, Veracruz se midieron los parámetros lbdm, lbco, dm y lt. Cada mazorca fue marcada con un número para su identificación y se tomó registro fotográfico de cada una. Este se realizó sobre un área color rojo con una cámara Fujifilm ${ }^{\circledR}$ de $12 \mathrm{MP}$ colocada de manera paralela al área de captura a 900 lúmenes.

Se realizó un procesamiento de cada imagen con el software Visual C 2010 y OpenCV 249 para determinar el área en pixeles. Se incluyó el área como un parámetro a evaluar con la variable A. Se localizó el centroide mediante la rutina Moment de OpenCV, luego se programó la localización del punto medio del lado tangente a la base, a partir del cual se determinó la variable de la distancia de la base al centroide (lbc) (Figura 2).

Los resultados de las mediciones obtenidas de la muestra de mazorcas variedad Murano que se obtuvieron se procesaron estadísticamente con el paquete Microsoft Excel versión 2013. El experimento tuvo como longitud total de la mazorca una mínima de $30 \mathrm{~cm}$, una máxima de 41.5 $\mathrm{cm}$, una media de $35.38 \mathrm{~cm}$, mientras que se ha reportado en pruebas longitudes máximas de 31.6 cm (Cruz-Meza et al., 2010), y la máquina señalada trabaja con mazorcas con un mínimo en longitud de $16 \mathrm{~cm}$. 


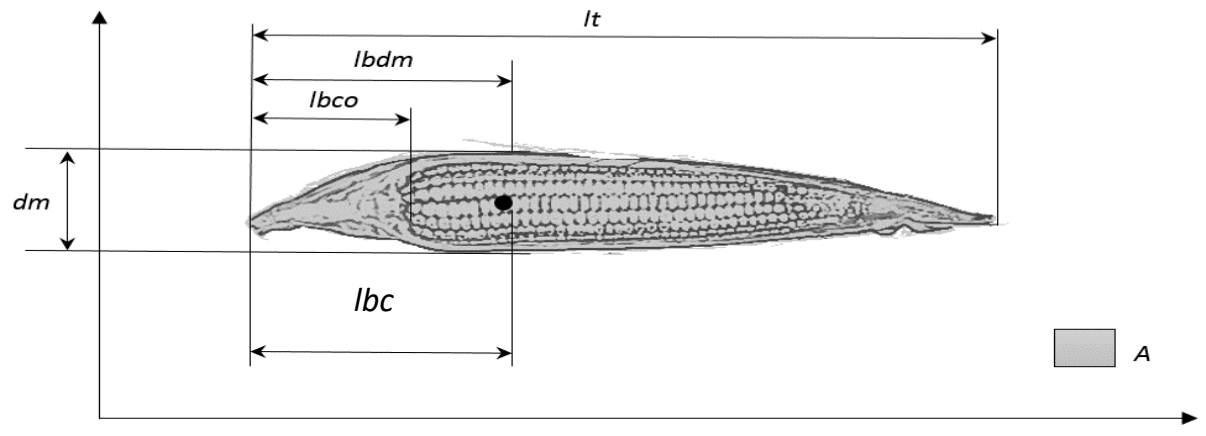

Figura 2. Parámetros geométricos medidos en mazorcas de la variedad Arroz.

De las diez combinaciones de dos variables, lbco se relacionó positivamente con la variable lbdm con una correlación de 0.91 (Cuadro 2) lo que indica que existe una fuerte relación entre estos dos parámetros.

Cuadro 2. Coeficiente de correlación de Pearson (r) para dos parámetros.

\begin{tabular}{|c|c|c|c|c|c|}
\hline \multicolumn{2}{|c|}{ Parámetros } & \multirow{2}{*}{$\frac{\mathrm{r}}{0.537^{\mathbb{I}}}$} & \multicolumn{2}{|c|}{ Parámetros } & \multirow{2}{*}{$\frac{\mathrm{r}}{0.021^{\mathbb{I}}}$} \\
\hline Do & $\mathrm{dm}$ & & do & lbco & \\
\hline Do & lbdm & $-0.125^{\mathbb{I}}$ & lbco & lt & $0.666^{\mathrm{dI}}$ \\
\hline lbco & $\mathrm{dm}$ & $-0.043^{\mathrm{II}}$ & $\mathrm{dm}$ & lbdm & $-0.049^{\text {II }}$ \\
\hline lbco & lbdm & 0.905 & $\mathrm{dm}$ & lt & $0.15^{\text {II }}$ \\
\hline do & $\mathrm{Lt}$ & $0.203^{\text {II }}$ & lbdm & lt & $0.6^{\mathrm{II}}$ \\
\hline
\end{tabular}

$\mathbb{\pi}=$ correlación no significativa.

De los parámetros de la combinación indicada se probó que existe una pendiente distinta de cero del modelo buscado. Se realizó un análisis de varianza teniendo en cuenta el valor Fisher de $\mathrm{F}_{\mathrm{n}, 0.05}^{\mathrm{m}}$, el estadístico $\mathrm{F}=216.39$ que se comparó contra $\mathrm{F}_{1,0.05}^{48}=4.04$. Como: $\mathrm{F}>\mathrm{F}_{\mathrm{n}}^{\mathrm{m}}$ se prueba que existe al menos un grado de correlación entre las variables. En esta prueba se encontró relación entre el sistema fruto-pedúnculo de una mazorca, mediante una regresión lineal se ajustaron los datos a una línea para encontrar la distancia óptima de corte:

$\mathrm{lbco}=\mathrm{A}_{1}+\mathrm{B}_{1} * \mathrm{lbdm}$

Donde: $\mathrm{A}_{1}=0.8738, \mathrm{~B}_{1}=-0.2384$.

Al evaluar la ecuación (4) se obtuvo un error absoluto $\epsilon_{1}=0.737 \mathrm{~cm}$. Para la composición con tres variables se realizaron diez combinaciones entre cinco variables obteniendo los coeficientes de correlación múltiple y de determinación (Cuadro 3). Al analizar el Cuadro 3 se nota que las combinaciones lbco-lbdm-lt y lbco-dm-lbdm tienen un coeficiente de correlación mayor a 0.9, por lo que se comprobó la existencia de un modelo mediante un análisis de varianza para cada una.

De la primera combinación se obtuvo el estadístico $\mathrm{F}=125.27$ que se comparó con $\mathrm{F}_{2,0.05}^{48}=3.19$. Como: 125.27> 3.19 se concluye que existe correlación entre las variables ensayadas. Al analizar los datos de la combinación lbco-dm-lbdm, se observa que $\mathrm{F}=105.94$. Dado que $105.9>\mathrm{F}_{1}^{48} 0.05$ existe evidencia experimental de que hay un grado de correlación entre los parámetros. 
Cuadro 3. Coeficiente de correlación múltiple $(R)$ y Coeficiente de determinación $\left(R^{2}\right)$ entre diez diferentes combinaciones con tres variables de cinco parámetros.

\begin{tabular}{|c|c|c|c|c|c|c|c|c|c|}
\hline \multicolumn{3}{|c|}{ Parámetros } & \multirow{2}{*}{$\frac{\mathrm{R}}{0.254^{\mathrm{I}}}$} & \multirow{2}{*}{$\frac{\mathrm{R}^{2}}{0.064}$} & \multicolumn{3}{|c|}{ Parámetros } & \multirow{2}{*}{$\frac{\mathrm{R}}{0.681^{\mathrm{II}}}$} & \multirow{2}{*}{$\frac{\mathrm{R}^{2}}{0.464}$} \\
\hline do & lt & lbco & & & lbco & $\mathrm{dm}$ & lt & & \\
\hline lbco & lt & do & $0.676^{\mathbb{I I}}$ & 0.457 & do & $\mathrm{dm}$ & lbdm & $0.545^{\mathbb{I}}$ & 0.297 \\
\hline do & lt & lbdm & $0.369^{\mathbb{q}}$ & 0.136 & lbco & $\mathrm{dm}$ & do & $0.067^{\mathbb{I}}$ & 0.004 \\
\hline lbco & lbdm & lt & 0.917 & 0.842 & do & $\mathrm{dm}$ & lt & $0.55^{\mathbb{I I}}$ & 0.303 \\
\hline do & $\mathrm{dm}$ & lbco & $0.538^{\mathbb{I}}$ & 0.289 & Lbco & $\mathrm{dm}$ & $\mathrm{lbdm}$ & 0.904 & 0.818 \\
\hline
\end{tabular}

II= correlación no significativa.

La regresión lineal se llevó a cabo para ambas composiciones y se ajustó cada superficie a los datos. De los coeficientes obtenidos por esta se establecieron dos modelos que se evaluaron mediante el análisis del error absoluto:

lbco $=\mathrm{A}_{2}+\mathrm{B}_{2} * \mathrm{lbdm}+\mathrm{C}_{2} * \mathrm{lt}$

Donde: $\mathrm{A}_{2}=-4.1894, \mathrm{~B}_{2}=0.7624, \mathrm{C}_{2}=0.1582$.

lbco $=\mathrm{A}_{3}+\mathrm{B}_{3} * \mathrm{lbdm}+\mathrm{C}_{3} * \mathrm{dm}$

Donde: $\mathrm{A}_{3}=-0.3329, \mathrm{~B}_{3}=0.8739, \mathrm{C}_{3}=0.0014$.

Se procedió a buscar una correlación entre 4 y 5 variables, pero se nota que no existe una correlación que supera los 0.9 , por lo que no se llevó a cabo la regresión múltiple ni el análisis de varianza. Los datos obtenidos manualmente de la muestra de 103 mazorcas, el registro fotográfico y el posterior procesamiento de las imágenes permitió su binarizacion extrayendo el color propio de las mazorcas convirtiéndolos en pixeles negros, el conteo de ellos determina el área (A) de la mazorca (Figura 3) integrándose como un dato estadístico más. El valor de A y lbc se manejaron en pixeles.

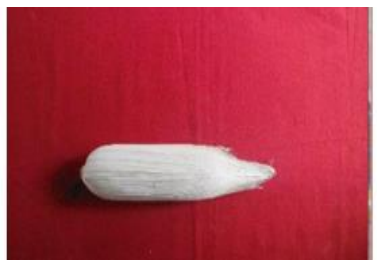

a)

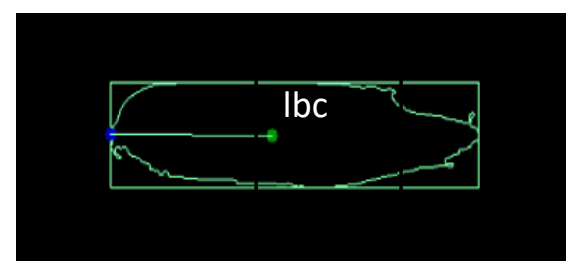

b)

Figura 3. Procesamiento de mazorcas en OpenCV para a) determinar el área y b) lbc.

Los valores medidos en pixeles presentan un error de \pm 30 pixeles por imagen procesada generado por el ruido presente en cada toma fotográfica. En el Cuadro 4 se nota que el largo máximo de esta variedad es similar a la de la variedad Murano, pero esta tiene una varianza mayor, esto se debe a que la variedad Arroz es criolla mientras que la primera es hibrida.

Esta variabilidad prevé una correlación menor entre las variables. Además, esta tiene una varianza de lbco de $1.6 \mathrm{~cm}$, comparada con los $4.9 \mathrm{~cm}$ de la muestra hibrida, la muestra criolla podría generar un algoritmo que no necesite un gran ajuste, por lo que se debe considerar para el diseño de máquinas deshojadoras de mazorcas. 
Cuadro 4. Estadística descriptiva de mazorcas de la variedad Arroz.

\begin{tabular}{|c|c|c|c|c|c|c|}
\hline \multirow{2}{*}{ Características } & lbco & lt & $\mathrm{lbdm}$ & $\mathrm{dm}$ & \multirow{2}{*}{$\frac{\mathrm{A}}{\text { (pixeles) }}$} & \multirow{2}{*}{$\frac{\mathrm{lbc}}{(\mathrm{mm})}$} \\
\hline & \multicolumn{2}{|c|}{$(\mathrm{cm})$} & \multicolumn{2}{|c|}{$(\mathrm{mm})$} & & \\
\hline Media & 4.49 & 33.1 & 90.94 & 58.33 & 26967 & 204.89 \\
\hline Mediana & 4.53 & 33 & 91.27 & 57.94 & 26731 & 201 \\
\hline Desviación estándar & 1.26 & 3.42 & 20.62 & 5.79 & 3970.25 & 28.49 \\
\hline Varianza de la muestra & 1.6 & 11.68 & 425.06 & 33.54 & 15762892.75 & 811.92 \\
\hline Mínimo & 1.07 & 25 & 42.95 & 42.91 & 17054 & 143 \\
\hline Máximo & 7.58 & 40 & 165.67 & 72.2 & 37312 & 290 \\
\hline
\end{tabular}

Al observar los datos de correlación del coeficiente de Pearson y el coeficiente de correlación múltiple para tres parámetros se notó que no existe una correspondencia significativa. En el Cuadro 5 se muestran los valores de los coeficientes de correlación múltiple para cuatro parámetros, de los cuales solo la combinación lbco, dm, lbdm y lbc cumple la condición de significancia. También es notorio que después de la variable lbdo, lbc cuenta con los valores de correlación más altos.

Cuadro 5. Coeficiente de correlación múltiple $(R)$ y coeficiente de determinación $\left(R^{2}\right)$ entre diez diferentes combinaciones con cuatro parámetros.

\begin{tabular}{cccccc}
\hline \multicolumn{2}{c}{ Parámetros } & & $\mathrm{R}$ & $\mathrm{R}^{2}$ \\
\hline Lbco & $\mathrm{dm}$ & $\mathrm{Lbdm}$ & $\mathrm{lt}$ & $0.637^{\mathrm{P}}$ & 0.406 \\
$\mathrm{lbco}$ & $\mathrm{dm}$ & $\mathrm{Lt}$ & $\mathrm{A}$ & $0.423^{\mathrm{P}}$ & 0.179 \\
$\mathrm{lbco}$ & $\mathrm{lbdm}$ & $\mathrm{Lt}$ & $\mathrm{A}$ & $0.624^{\mathrm{P}}$ & 0.389 \\
$\mathrm{lbco}$ & $\mathrm{dm}$ & $\mathrm{Lbdm}$ & $\mathrm{A}$ & $0.635^{\mathrm{P}}$ & 0.403 \\
$\mathrm{lbco}$ & $\mathrm{dm}$ & $\mathrm{Lbdm}$ & $\mathrm{lbc}$ & 0.657 & 0.431 \\
$\mathrm{lbco}$ & $\mathrm{lt}$ & $\mathrm{A}$ & $\mathrm{lbc}$ & $0.471^{\mathrm{P}}$ & 0.222 \\
$\mathrm{lbco}$ & $\mathrm{lbdm}$ & $\mathrm{A}$ & $\mathrm{lbc}$ & $0.642^{\mathrm{P}}$ & 0.412 \\
$\mathrm{lbco}$ & $\mathrm{dm}$ & $\mathrm{A}$ & $\mathrm{lbc}$ & $0.475^{\mathrm{P}}$ & 0.225 \\
$\mathrm{lbco}$ & $\mathrm{dm}$ & $\mathrm{Lt}$ & $\mathrm{lbc}$ & $0.493^{\mathrm{P}}$ & 0.243 \\
$\mathrm{lbco}$ & $\mathrm{lbdm}$ & $\mathrm{Lt}$ & $\mathrm{lbc}$ & $0.641^{\mathrm{P}}$ & 0.41 \\
\hline
\end{tabular}

$\mathrm{P}=$ correlación no significativa.

Al realizar el análisis de varianza se comparó el estadístico $\mathrm{F}_{3,0.05}^{102}=2.69$ con $\mathrm{F}=25.05$, dado que $\mathrm{F}>\mathrm{F}_{3,0.05}^{102}$ se tiene que existe un grado de correlación entre las variables a lo que se realizó una regresión lineal. De la regresión lineal múltiple se obtuvo la siguiente ecuación:

lbco $=\mathrm{A}_{4}+\mathrm{B}_{4} * \mathrm{dm}+\mathrm{C}_{4} * \mathrm{lbdm}+\mathrm{D}_{4} * \mathrm{lbc}$

Donde: $\mathrm{A}_{4}=-1.92, \mathrm{~B}_{4}=0.03, \mathrm{C}_{4}=0.03$ y $\mathrm{D}_{4}=0.01$.

Esta ecuación se evaluó y obtuvo $\epsilon_{4}=0.755 \mathrm{~cm}$ que comparada con la ecuación (6) tienen una diferencia de $0.018 \mathrm{~cm}$ en el error absoluto evaluado. Para la combinación de cinco parámetros. En el Cuadro 6 dos composiciones tienen un coeficiente de correlación múltiple mayor a 0.65, por lo que ambas se analizaron mediante el análisis de varianza. Se sabe que $F>F_{4,0.05}^{102}=2.46$, comprobando la existencia de una correlación significante entre los parámetros, de modo que se realizó una regresión múltiple para cada combinación. 
Cuadro 6. Coeficiente de correlación múltiple $(R)$ y coeficiente de determinación $\left(R^{2}\right)$ entre cuatro diferentes combinaciones con cinco parámetros.

\begin{tabular}{lcccccc}
\hline & \multicolumn{2}{c}{ Parámetros } & & & $\mathrm{R}$ & $\mathrm{R}^{2}$ \\
\hline $\mathrm{lbco}$ & $\mathrm{dm}$ & $\mathrm{lbdm}$ & $\mathrm{lt}$ & $\mathrm{A}$ & $0.637^{\mathrm{P}}$ & 0.406 \\
$\mathrm{lbco}$ & $\mathrm{dm}$ & $\mathrm{lbdm}$ & $\mathrm{lt}$ & $\mathrm{lbc}$ & $0.657^{\mathrm{b}}$ & 0.432 \\
$\mathrm{lbco}$ & $\mathrm{dm}$ & $\mathrm{Lt}$ & $\mathrm{A}$ & $\mathrm{lbc}$ & $0.499^{\mathrm{P}}$ & 0.249 \\
$\mathrm{lbco}$ & $\mathrm{lbdm}$ & $\mathrm{Lt}$ & $\mathrm{A}$ & $\mathrm{lbc}$ & $0.643^{\mathrm{P}}$ & 0.413 \\
$\mathrm{lbco}$ & $\mathrm{dm}$ & $\mathrm{lbdm}$ & $\mathrm{A}$ & $\mathrm{lbc}$ & 0.662 & 0.438 \\
\hline
\end{tabular}

$\mathrm{P}=$ Correlación no significativa.

De la regresión lineal múltiple se obtuvieron las siguientes ecuaciones:

lbco $=A_{5}+B_{5} * d m+C_{5} * \mathrm{lbdm}+D_{5} * A+E_{5} * \mathrm{lbc}$

Donde: $\mathrm{A}_{5}=-2.32, \mathrm{~B}_{5}=0.05, \mathrm{C}_{5}=0.03, \mathrm{D}_{5}=0.00$ y $\mathrm{E}_{5}=0.01$.

$\mathrm{lbco}=\mathrm{A}_{6}+\mathrm{B}_{6} * \mathrm{dm}+\mathrm{C}_{6} * \mathrm{lbdm}+\mathrm{D}_{6} * \mathrm{lt}+\mathrm{E}_{6} * \mathrm{lbc}$

Donde: $\mathrm{A}_{6}=-1.74, \mathrm{~B}_{6}=0.03, \mathrm{C}_{6}=0.03, \mathrm{D}_{6}=-0.01 \mathrm{y} \mathrm{E}_{6}=0.01$.

$\mathrm{Al}$ evaluar las igualdades se encontró para (8) un error $\epsilon_{5}=0.761$, para (9) un error $\epsilon_{6}=0.759$. En el Cuadro 7 se observa que la combinación de seis parámetros también cumple la condición, a lo que se llevó a cabo el análisis de varianza. Esta combinación presenta además el valor más alto de correlación múltiple.

Cuadro 7. Coeficiente de correlación múltiple $(R)$ y coeficiente de determinación $\left(R^{2}\right)$ con seis parámetros.

\begin{tabular}{cccccccc}
\hline \multicolumn{3}{c}{ Parámetros } & & $\mathrm{R}$ & $\mathrm{R}^{2}$ \\
\hline lbco & $\mathrm{dm}$ & $\mathrm{lbdm}$ & lt & $\mathrm{A}$ & $\mathrm{lbc}$ & 0.662 & 0.438 \\
\hline
\end{tabular}

Se comparó el estadístico $\mathrm{F}_{5,0.05}^{102}=2.3$, con $\mathrm{F}$, evidenciando que $\mathrm{F}>F_{5,0.05}^{102}$, así que se prosiguió a realizar la regresión lineal. Se obtuvo la siguiente ecuación, que una vez evaluada se valoró el error $\epsilon_{7}=0.757$, que es mayor que el error de (9).

lbco $=\mathrm{A}_{7}+\mathrm{B}_{7} * \mathrm{dm}+\mathrm{C}_{7} * \mathrm{lbdm}+\mathrm{D}_{7} * \mathrm{lt}+\mathrm{E}_{7} * \mathrm{~A}+\mathrm{F}_{7} * \mathrm{lbc}$

Donde: $\mathrm{A}_{7}=-2.62, \mathrm{~B}_{7}=0.05, \mathrm{C}_{7}=0.03, \mathrm{D}_{7}=0.02, \mathrm{E}_{7}=0.00 \mathrm{y} \mathrm{F}_{7}=0.01$.

Aunque la variedad Murano presentó los valores más altos de correlación, su uso para comercio de hojas es mínimo, en las zonas productoras de maíz para hoja, se cultivan variedades criollas con un alto índice de variabilidad en su tamaño. Esta particularidad hace que la ecuación (10) sea la más conveniente para usarse en un entorno de programación considerando que el error absoluto en la distancia de corte es de menos de $8 \mathrm{~mm}$ en todas las ecuaciones.

\section{Conclusiones}

El análisis de los parámetros geométricos demostró que existe una relación entre los parámetros medidos de las mazorcas que permite determinar la longitud de la base al diámetro de corte óptimo mediante modelos matemáticos que varían según las variedades, Murano y Arroz. Se desarrollaron los algoritmos para calcular dos parámetros más a considerar en el análisis, área y longitud de la base al centroide, mediante el uso del software OpenCV. 
A partir, de los datos experimentales se establecieron tres modelos lineales para calcular la longitud de la base al diámetro de corte óptimo en una variedad hibrida uniforme de maíz de la variedad Murano. También se generaron cuatro modelos para determinar la longitud de la base al diámetro de corte óptimo en una variedad criolla de maíz Arroz.

Es posible usar estos modelos para calcular la longitud de corte idónea en mazorcas de maíz de la variedad Murano y Arroz. Dado que la productividad de una máquina deshojadora de mazorcas para tamal es nueve veces superior a la manual, el aplicar estas ecuaciones en un sistema de control automático para mejorar dicha maquinaria, incrementaría la eficiencia de la cosecha obteniendo mejores resultados en calidad de hojas y un menor desperdicio de grano.

\section{Literatura citada}

Ávila, M.; Borges, E. O. L. y Bernaez, J. C. 2009. Caracterización biométrica del cultivo y perfil descriptivo de mazorcas del hibrido de maíz superdulce 'delicia' (BT1). Bioagro. 21(2):143-147.

Bernabe, E.; Romatchick, E.; Cebada, J. and Velazquez, N. 2018. Design of a position control for a cutting system of a maize-husking machine. IEEE Latin America Transactions. 16(4):22-29.

Bouza, M. Y.; Martínez, R., A.; Mederos, L. D. y Pérez, B. P. 2006. Determinación de propiedades físico-mecánicas de los frutos del Nim relacionadas con la cosecha mecanizada por vibración. Ciencias Técnicas Agropecuarias. 15(3):22-27.

Castañeda Zavala, Y. 2007. Una visión sobre la importancia de la diversidad del maíz en México. Christus. 72(762:20-22.

Cruz-Meza, P.; Romantchik-Kriuchkova, E. and Hahn-Schlam, F. F. 2010. Design, construction, and evaluation of a husking machine to permit the use of the husks as wrapping for tamales. Ingeniería Agrícola y Biosistemas. 2(2):69-74.

Hahn-Schlam, F. F.; Martínez-Díaz, P. y Reyes-Amador, A. 2012. Modelo teórico para predecir el lugar del corte y optimizar la extracción de la pulpa en coco. Ingeniería Agrícola y Biosistemas. 4(1):13-17.

Infante, G. S. y Zárate de Lara, G. P. 1984. Métodos estadísticos: un enfoque interdisciplinario. 1 (Ed.). Trillas. 401-463.

Ortiz-Torres, E.; Antonio, P.; Gil-Muñoz, A.; Guerrero-Rodríguez J. D.; López-Sánchez H.; Taboada-Gaytán, O. R.; Hernández-Guzmán, J. A. y Valadez-Ramírez M. 2013. Rendimiento y calidad de elote en poblaciones nativas de maíz de Tehuacán, Puebla. Rev. Chapingo Ser. Hortic. 19(2):225-238.

Pérez-López, A.; Villaseñor-Perea, C. A.; Crisanto-Martínez, V. y Corrales-García, J. J. E. 2009. Propiedades mecánicas y maduración de frutos de mango (Mangifera indica L.) bajo compresión axial. Ingeniería Agrícola y Biosistemas. 1(1):19-23.

Prado-Martínez, M; Anzaldo-Hernández, J.; Becerra-Aguilar, B.; Palacios-Juárez, H.; VargasRadillo, J.J. y Rentería-Urquiza, M. 2012. Caracterización de hojas de mazorca de maíz y de bagazo de caña para la elaboración de una pulpa celulósica mixta. Madera y Bosques. 18(3):37-51. 